\title{
Major histocompatibility complex variation and age-specific endoparasite load in subadult European rabbits
}

\author{
CLAUS OPPELT,${ }^{*}+$ ANETT STARKLOFF,$*$ PHILIPP RAUSCH,$*$ DIETRICH VON HOLST* and \\ HEIKO G. RÖDEL* \\ *Department of Animal Physiology, University of Bayreuth, D-95440 Bayreuth, Germany, tLimnological Institute, University of \\ Konstanz, D-78464 Konstanz, Germany
}

\begin{abstract}
Genes of the major histocompatibility complex (MHC) play a fundamental role in the vertebrate immune response and are amongst the most polymorphic genes in vertebrate genomes. It is generally agreed that the highly polymorphic nature of the MHC is maintained through host-parasite co-evolution. Two nonexclusive mechanisms of selection are supposed to act on MHC genes: superiority of MHC heterozygous individuals (overdominance) and an advantage for rare MHC alleles. However, the precise mechanisms and their relative importance are still unknown. Here, we examined MHC dependent parasite load in European rabbits (Oryctolagus cuniculus) from a distinct population with low MHC diversity (three alleles, six genotypes). Using a multivariate approach, we tested for associations of individual MHC class II DRB constitution and the rabbits' intestinal burden with nematodes and coccidia. Rabbits having a particular allele showed lower infestations with hepatic coccidia (E. stiedai). However, a comparison of all six genotypes in the population revealed that carriers of this allele only benefit when they are heterozygous, and furthermore, MHC heterozygosity in general did not affect individual parasite load. In conclusion, this study suggests an immunogenetic basis of European rabbit resistance to hepatic coccidiosis, which can strongly limit survival to maturity in this species. Our study gives a complex picture of MHC-parasite correlations, unveiling the limits of the classical hypotheses of how MHC polymorphism is maintained in natural systems.
\end{abstract}

Keywords: disease, immunogenetics, MHC, Oryctolagus cuniculus, overdominance, parasite burden

\section{Introduction}

The major histocompatibility complex (MHC) of higher vertebrates is an exceptionally gene-dense genomic region with more than one hundred genes predominantly expressing immune-related proteins (The MHC Sequencing Consortium 1999). The so-called classical (class I and class II) MHC genes play a central role in vertebrate adaptive immunity. They code for proteins that present short peptides on the surface of cells. An

Correspondence: Heiko G. Rödel, Tel.: 0049921 552484; Fax: 0049921 552794; E-mail: heiko.roedel@uni-bayreuth.de immune response is triggered when an MHC non-selfpeptide complex is recognized by a $\mathrm{T}$ cell (Janeway et al. 2001). Parasites might therefore adapt to escape this recognition system by avoiding the expression of structures that can be bound by MHC molecules. This leads to an ongoing co-evolution of defence and escape mechanisms of hosts and parasites and might explain why classical MHC genes are by far the most polymorphic genes within vertebrate genomes (Apanius et al. 1997).

Two nonexclusive mechanisms of natural selection have been proposed to maintain this polymorphism. On the one hand, the negative frequency-dependent 
selection hypothesis states that rare allelic variants confer an advantage to their carriers, because parasites probably adapt to the most frequent alleles of the host population (Takahata \& Nei 1990). Within a population, this mode of selection would result in an association of particular alleles with low or high susceptibility to some parasites. On the other hand, the occurrence of particular combinations of alleles could lead to a higher parasite resistance, i.e. heterozygosity (Doherty \& Zinkernagel 1975) or the possession of two divergent alleles (Wakeland et al. 1990). MHC genes are codominantly expressed, so individuals with different alleles at each MHC locus are able to present a greater variety of peptides and therefore can trigger an immune response against a wider spectrum of parasites (Doherty \& Zinkernagel 1975). Finally, the two selective modes, rare allele advantage and heterozygote superiority (overdominance, McClelland et al. 2003), may interact in several ways. For example, rare alleles are unlikely to appear in homozygous individuals and the heterozygous combination of two disadvantageous alleles will obscure any advantage provided by heterozygosity.

To date, supporting and contradicting findings give a complex picture of these mechanisms. Several studies have shown the association of single MHC alleles with susceptibility or resistance to different diseases and parasites in a variety of vertebrate species (e.g. Paterson et al. 1998; Harf \& Sommer 2005; Westerdahl et al. 2005; Croisetière et al. 2008; Tollenaere et al. 2008). Also, evidence has been provided that MHC heterozygosity lowers the susceptibility to infections with single or multiple parasite species (e.g. Arkush et al. 2002; Froeschke \& Sommer 2005; Westerdahl et al. 2005; Kekäläinen et al. 2009; but see Wedekind et al. 2005; Ilmonen et al. 2007). But some studies indicate that instead of heterozygosity per se only particular combinations of alleles with divergent nucleotide sequences are advantageous (Thursz et al. 1997; Ditchkoff et al. 2001; Richman et al. 2003).

Excess polymorphism is the outstanding feature of classical MHC genes. Thus, the majority of populations studied for associations between $\mathrm{MHC}$ and parasites show high MHC variability, and as a consequence, the number of study animals with particular MHC alleles or particular combinations is usually low. On the one hand, this can substantially lower the chance of detecting existing MHC-related effects. On the other hand, finding and testing a large number of traits in only a few animals increases the risk of obtaining false positives. Consequently, studies on populations with moderate numbers of MHC genotypes and thus occurring at higher frequencies could contribute significantly to our knowledge of the mechanisms driving the association between MHC constitution and an animal's resis- tance to parasites (Kekäläinen et al. 2009; Oliver et al. 2009), although such a situation might only rarely occur in natural populations (but see e.g. Babik et al. 2005).

Some parasites (in a broad sense including viruses, pro- and eukaryotic organisms feeding on the resources of their host) trigger lethal diseases or are cleared by the hosts' immune system. Other parasites threaten the host, thereby lowering its fitness by reducing fecundity and body condition. For example, intestinal nematodes and protozoans like plasmodium and coccidia rather belong to the latter group. Such endoparasites are particularly interesting, because they are usually common in host populations and often challenge their hosts throughout their lives (Donald et al. 1982). Therefore, the selective pressure on adaptations to such endoparasites should be high.

Here, we investigated the relationship between MHC and endoparasite burden in European rabbits (Oryctolagus cuniculus). As in most mammals, the MHC class II region of the rabbit was found to contain several expressed alpha and beta genes (LeGuern et al. 1985; Sittisombut \& Knight 1986; Sittisombut et al. 1989; Spieker-Polet et al. 1989). More recent studies use rabbit MHC genes for population genetic analysis but focus on the gene DQA (Fain et al. 2001; Goüy de Bellocq et al. 2008; Surridge et al. 2008), while emphasis in most other mammals is on the DRB genes (Sommer 2005). We examined the association between the individual MHC class II DRB constitution and the endoparasite burden of animals from a European rabbit population living in a field enclosure. We focused on subadults because premature mortality in the European rabbit is very high (Richardson \& Wood 1982; von Holst et al. 2002; Rödel et al. 2004, 2008b, 2009) and endoparasites are frequently considered a critical factor for juvenile survival in this species (Mykytowycz 1956; Gibb 1979; Cowan 1987). In particular, we tested for heterozygote superiority and advantage of specific alleles and genotypes with respect to endoparasite burden using a multivariate approach controlling for potentially confounding effects. We focused on the most common species of endoparasites hosted by European rabbits: the intestinal nematodes Graphidium strigosum in the stomach, Trichostrongylus retortaeformis colonizing the small intestine and Passalurus ambiguus predominantly occurring in the caecum and colon, and different coccidia (Eimeria) species infesting the liver and the gut. All of these endoparasites can cause severe disease such as diarrhoea and anaemia leading to body mass loss, in particular in young animals, and especially Eimeria species are known to be lethal when the animals show high infestations with these parasites (Hobbs et al. 1999; Pakandl 2009). 


\section{Material and methods}

\section{Study population}

The study was conducted on animals from a fenced European rabbit population living in a $20000 \mathrm{~m}^{2}$ enclosure situated next to the campus of the University of Bayreuth (Franconia, Germany). The population consisted of descendants of animals that had been caught in the wild (Bavaria, Germany) in 1983. During the study period (2006-2007), the population consisted of about 50 adult individuals with a sex ratio of about two females to one male. In the past, the population underwent two bottlenecks because of high winter mortality in one year and because of an outbreak of myxomatosis some years later. For this reason, the overall genetic and $\mathrm{MHC}$ variability was expected to be low.

Vegetation consisted of grassland interspersed with groups of trees and bushes, which represents an adequate habitat structure for the European rabbit (Corbet 1994). In addition to the burrows and breeding stops dug by the rabbits (around 40-50), the area contained 16 artificial concrete warrens with interconnected chambers and removable tops. These were used by the rabbits as the main warrens of their group territories and for breeding (more details in von Holst et al. 2002; Rödel et al. 2004).

\section{Study animals}

We conducted our study on young rabbits of known age and origin. To this end, we daily checked for newborn litters during the breeding season 2006 and 2007 from mid March to late September (see Rödel et al. 2008a). We prepared all natural warrens and breeding stops dug by the animals with artificial vertical openings to the nest chambers, which we covered with concrete flagstones. Therefore, we could record the birth of all young rabbits within $24 \mathrm{~h}$. We recorded litter size, and 12 days later (about 1 week before the young rabbits leave their breeding burrow), we determined the sex and marked the pups individually with a numbered plastic tag (Dalton Rototag, $20 \times 5 \times 1 \mathrm{~mm}$, $0.25 \mathrm{~g}$; Dalton Continental) in one ear. When the animals reached a body mass of about $1000 \mathrm{~g}$, we trapped them (by wooden live traps baited with salted peanuts), removed the small plastic tag and replaced it with a coloured aluminium tag $(45 \times 20 \mathrm{~mm}, 1.1 \mathrm{~g})$ fixed with a plastic tag (Dalton Rototag, $35 \times 10 \times$ $2 \mathrm{~mm}, 1.5 \mathrm{~g}$ ).

In late autumn (early November) in 2006 and 2007, we captured and sacrificed a total of 113 young rabbits, which were between 54 and 248 days old $\left(n_{2006}=43\right.$; $n_{2007}=70$ ). We took tissue samples from the ears and stored them in $70 \%$ ethanol at $4{ }^{\circ} \mathrm{C}$. Spleen tissue was stored in RNA stabilization reagent (RNAlater, Qiagen) at $-20{ }^{\circ} \mathrm{C}$. The carcasses were packed individually in plastic bags and frozen at $-15{ }^{\circ} \mathrm{C}$ until they were dissected for the quantification of their endoparasite load.

\section{Determination of endoparasite load}

Nematodes. Infestations with nematodes in the gastrointestinal tract were quantified by the total number of adult worms per individual rabbit. For this, the contents of the abdominal cavity were removed from the defrosted animals. The alimentary tract was separated into four regions: stomach, small intestine, caecum and colon. The content of each part was placed in separate beakers and diluted with water to $1 \mathrm{~L}$, and the numbers of G. strigosum, T. retortaeformis and P. ambiguus were recorded. For this, we repeatedly poured the dilution into a flat bowl (about $20 \mathrm{~mL}$ content) with a grid pattern (1 cm squares) and counted the different species of nematodes using a low-power dissection microscope. We only considered adult worms. For quantifying the number of nematodes in the stomach (G. strigosum), we used the complete diluted stomach content $(1 \mathrm{~L})$. T. retortaeformis (colonizing the small intestine) and $P$. ambiguus (occurring in caecum and colon) were quantified using samples of $100-200 \mathrm{~mL}(10-20 \%)$ from the dilutions of the content from the small intestine, caecum and colon. We then extrapolated the counts to the total amount of $1 \mathrm{~L}$. This was carried out owing to the very high worm abundance in these parts of the gut.

Coccidia. Infestations with coccidia (Eimeria) were quantified by the occurrence of coccidia oocytes per gram of faeces. To this end, we removed three faecal pellets from the colon (about $0.5 \mathrm{~g}$ ) and stored them in plastic vials at $-15^{\circ} \mathrm{C}$ until analysis. We then diluted the pellets in a flotation solution $\left(307.7 \mathrm{~g} \mathrm{MgSO}_{4}\right.$ in $500 \mathrm{~mL}$ $\mathrm{H}_{2} \mathrm{O}$ ) and counted the oocytes in a modified McMaster chamber (Gordon \& Whitlock 1939). We then calculated the number of oocytes per gram of faeces.

For a more detailed determination of the different Eimeria species, we placed about $5 \mathrm{~mL}$ of the pellet dilution in a small bin. After $30 \mathrm{~min}$, a cover slip was placed briefly on the surface of the solution and the floated oocytes attached to this cover slip were examined under a microscope. We counted 100 oocytes and classified them in oocytes from Eimeria stiedai (infesting the liver; oocytes are excreted via faeces) and other oocytes from the genus Eimeria occurring in the gut. For classification, we followed the descriptions of Kessel \& Jankiewicz (1931) and Kotlan \& Pellerdy (1949). The proportion of the two Eimeria classes was multiplied by 
the total number of eggs so as to estimate the total amount of hepatic and gut coccidia oocytes.

\section{MHC DRB genotyping and expression}

DNA was extracted using DNeasy Tissue Kit (Qiagen) following the manufacturer's instructions from tissue samples taken from the ear and stored in $70 \%$ ethanol. To conduct PCR, we used the primers JS1 and JS2 (Schad et al. 2004), which have been developed for lemurs, but also have been successfully used in rodents (e.g. Babik et al. 2005; Oliver \& Piertney 2006). These primers amplify a $171 \mathrm{bp}$ fragment (excluding primers) of the MHC class II DRB exon 2 containing most of the polymorphic antigen-binding site. PCR was carried out in $20 \mu \mathrm{L}$ reaction volumes with $1 \mu \mathrm{L}$ extracted genomic DNA, 1× PCR reaction buffer with $1.5 \mathrm{~mm} \mathrm{MgCl}_{2}$, $0.4 \mu \mathrm{M}$ of each primer, $140 \mu \mathrm{M}$ of each dNTP and $0.5 \mathrm{U}$ Taq polymerase (Genecraft). Cycling conditions were as follows: $5 \mathrm{~min}$ at $94^{\circ} \mathrm{C}, 30$ cycles of $1 \mathrm{~min}$ at $94{ }^{\circ} \mathrm{C}$, $2 \mathrm{~min}$ at $55^{\circ} \mathrm{C}, 3 \mathrm{~min}$ at $72{ }^{\circ} \mathrm{C}$ and a final elongation step of $7 \mathrm{~min}$ at $72{ }^{\circ} \mathrm{C}$.

For DRB genotyping, a combined single-strand conformation polymorphism and heteroduplex analysis (SSCP/HD) was conducted. Five microlitres of each PCR product were mixed with an equal volume of formamide buffer ( $95 \%$ formamide), denatured for $5 \mathrm{~min}$ at $96{ }^{\circ} \mathrm{C}$ on a robocycler and immediately chilled on ice water for $10 \mathrm{~min}$. The mixture was carried on a $9 \%$ nondenaturing polyacrylamide gel (37.5:1) cooled to $10{ }^{\circ} \mathrm{C}$. Electrophoresis was carried out for $3 \mathrm{~h}$ with $8 \mathrm{~W}$ per gel (Maxigel, Biometra). Under these conditions, denatured DNA forms double-stranded heteroduplices (HD) and single-stranded conformations (SSCP). Both are sequence dependent and thus allow discriminating different genotypes. Gels were silver-stained after electrophoresis (Budowle et al. 1991). Samples with equal banding patterns were rearranged and run again on a nondenaturing PAGE on neighbouring lanes to enable the genotyping. From at least two individuals of each genotype, all SSCP-Bands were cut out and incubated in $80 \mu \mathrm{L}$ water for at least $3 \mathrm{~h}$ at $37^{\circ} \mathrm{C}$. One microlitre was used as template in another PCR according to the conditions described before. Again, products were screened by SSCP analysis, and every unique allele was sequenced (Seqlab, Göttingen, Germany) in both directions. Sequences were aligned using Bioedit 7.0.9 (Hall 1999); $d_{N}, d_{S}$ and allelic distances were calculated with Mega4 (Tamura et al. 2007).

Spleen samples of 12 rabbits (two of each genotype) were used to examine the expression of alleles. RNA was extracted (InnuPrep RNA mini kit, Analytic Jena), and genomic DNA was removed using DNAse (Fermentas). cDNA synthesis was carried out with reverse transcriptase and oligo(dT) $)_{18}$ primer (Fermentas) in 20$\mu \mathrm{L}$ reaction volumes with incubation at $37^{\circ} \mathrm{C}$ for $60 \mathrm{~min}$ and inactivation at $70^{\circ} \mathrm{C}$ for $5 \mathrm{~min}$. The obtained cDNA was used as a template in a further PCR. DNAse-digested RNA solutions served as negative controls to ensure the full elimination of genomic DNA. Amplificates from cDNA templates were carried on a nondenaturing gel for SSCP/HD analysis. Bands were cut out, reamplified and sequenced. Additionally, amplificates were sequenced directly when the SSCP/HD banding pattern indicated only a single allele.

The quantification of endoparasite load and MHC genotyping was conducted by different groups of investigators blind to each others' results.

\section{Statistical analyses}

The main focus of this study was to test the effects of parameters of the animals' MHC constitution together with several other individual characteristics on the load with different endoparasite species (response variables). This was performed with multivariate models (linear mixed effects models that allow to adjust for repeated measurements) using the statistic software $R$ version 2.10.1 ( $\mathrm{R}$ Development Core Team 2009). For this, we used the lme4 package (Bates 2005). The program $R$ does not directly provide $P$-values for mixed effects models calculated with lme4. Thus, we extracted the $P$ values and also the parameter estimates by Markovchain Monte Carlo sampling based on 10000 simulation runs (Baayen et al. 2008).

In a first step (see Table 2), we tested the effects of absence/presence of the different alleles (OrcuDRB*02, $\left.\operatorname{OrcuDRB} B^{*} 03, \operatorname{OrcuDR} B^{*} 04\right)$ and thus included them as fixed factors with two levels. In addition, we included heterozygosity (yes/no) as a fixed factor with two levels. Year was also included as a fixed factor (with two levels) to correct for differences in the environmental conditions between years, which might have potentially affected the spreading of endoparasites within the population. In addition, we included litter identity as a random factor, because several of the animals were litter siblings ( $n=113$ young rabbits from 56 litters) born to 28 different mothers. Litter mates can be expected to share a genetic background and experience similar conditions during their early development, which might have affected their probability of infestation with parasites in a similar way. We checked for potential dependencies (collinearities) among the occurrence of the different alleles and heterozygosity (Table 2). This was carried out with logistic regression models where we pairwise checked the correlations between the different variables and we assessed the explained variation with 
Nagelkerke's $R^{2}$ (Nagelkerke 1991). The results revealed marginal $\left(D R B^{*} 02\right.$ vs. heterozygosity: $R_{\text {Nagelkerke }}^{2}=0.009$; $D R B^{*} 03$ vs. $D R B^{*} 04: R_{\text {Nagelkerke }}^{2}=0.017$ ) or moderate correlations between the different predictor variables $\left(D R B^{*} 04\right.$ vs. heterozygosity: $R_{\text {Nagelkerke }}^{2}=0.184 ; D R B^{*} 02$ vs. $D R B^{*} 03: R_{\text {Nagelkerke }}^{2}=0.241 ; D R B^{*} 03$ vs. heterozygosity: $\quad R_{\text {Nagelkerke }}^{2}=0.264 ; \quad D R B^{*} 02$ vs. $D R B^{*} 04$ : $R_{\text {Nagelkerke }}^{2}=0.321$ ); thus, we concluded that there were no serious problems with collinearities among these predictor variables. In addition, we checked for multicollinearities among all predictor variables (including age, sex and year) by calculating variance inflation factors (VIF) for each model as given in Table 2. All VIF values were lower than three, indicating no problems with multicollinearities within the models (Fox \& Monette 1992).

In a second step, we tested for differences between the different genotypes, i.e. the different combinations of OrcuDRB alleles, on endoparasite load. For this, we used genotype as a fixed factor with six levels (see Fig. 4). Again, we included year and sex as predictor variables and litter identity as a random factor. Post hoc comparisons between the different levels of genotype were calculated by applying the same mixed model structure to the respectively reduced data set.

In both steps, we tested all 2-way interactions with sex or year. Nonsignificant interaction terms $(P>0.05)$ were stepwise reduced, and the models were recalculated. Response variables (different measures of parasite load) of the different models were $\log (x), \log (x+0.5)$ (in case the data set included zeroes) or $x^{0.2}$-transformed to adjust them to a normal distribution. Normality of the model residuals was checked visually by normal probability plots and with the Shapiro-Wilk test, and we assured the homogeneity of variances and goodness of fit by plotting residuals versus fitted values (Faraway 2006).

Deviation of MHC allele frequency distribution from Hardy-Weinberg equilibrium was tested using a Markov-chain (Guo \& Thompson 1992) in Arlequin 3.11 (Excoffier et al. 2005). The number of synonymous and nonsynonymous substitutions as well as Z-tests for directed selection was calculated in Mega4 (Tamura et al. 2007) using the method of Nei-Gojobori (1986).

\section{Results}

\section{MHC variability and expression of alleles}

In 113 genotyped subadult rabbits of the study population, we found only six different SSCP/HD banding patterns representing six different MHC DRB genotypes. By sequencing, we identified one to three alleles per genotype, indicating that the primers used here amplified more than one locus in some of the animals. But also the primer system amplified only a subset of the five DRB genes determined by Sittisombut et al. (1989). In total, we identified four different alleles and labelled them following the nomenclature of Klein et al. (1990): $\operatorname{OrcuDRB}{ }^{*} 01$ to $\operatorname{OrcuDRB}{ }^{*} 04$ (accession numbers GQ168795-GQ168798, Fig. 1). OrcuDRB*02 is identical to a previously published DRB1 sequence of the domestic rabbit (accession number EU427425).

Because MHC variability in the study population was fairly low, it was possible to reconstruct the constitution of haplotypes by applying Mendelian rules. At least three orthologous alleles or haplotypes $(a, b, c)$ are needed to form six genotypes ( $a a, b b, c c, a b, a c, b c$ ). Because in some genotypes more than one gene was typed, one of the four alleles has to be located on a second locus. Any other distribution of alleles (two loci with two alleles each; three loci, one with two, two with one allele) would result in only two haplotypes because DRB genes are tightly linked. But two haplotypes are insufficient to form six genotypes. $D R B^{*} 02,{ }^{*} 03$ and ${ }^{*} 04$ are not linked, because each of them appears without the other two in one genotype each. Furthermore, we never found more than two of these three alleles per individual. Thus, they represent a distinct locus or at least perfectly behave as if they were orthologous. $D R B^{*} 02$ and ${ }^{*} 03$ never appear without $D R B^{*} 01$, so that this allele is present at an additional locus on the haplo-

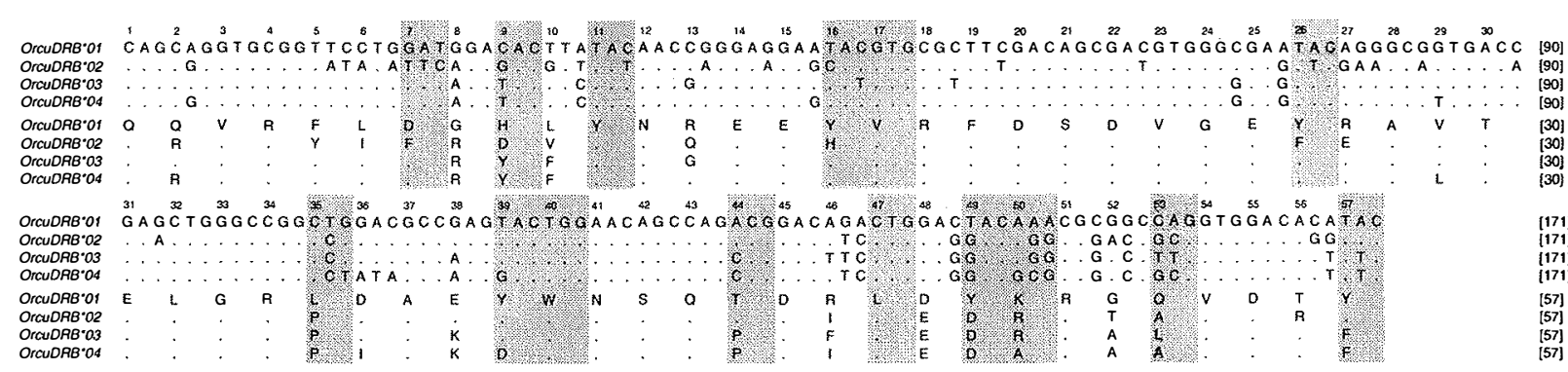

Fig. 1 Nucleotide and amino acid sequences of the alleles $\operatorname{OrcuDRB} B^{*} 01,{ }^{*} 02,{ }^{*} 03,{ }^{*} 04$. Sites of the putative peptide-binding region according to Brown et al. (1993) are shaded grey. 


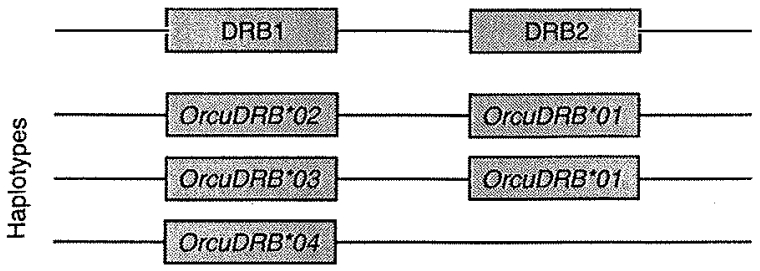

Fig. 2 Three occurring haplotypes in subadults of the European rabbit study population. DRB1 and DRB2 represent two distinct loci in hypothetical order.

type carrying $D R B^{*} 02$ as well as on the haplotype carrying $D R B^{*} 03$. Therefore, individuals with the two alleles $D R B^{*} 01^{*} 02$ or $D R B^{*} 01^{*} 03$ are homozygous. This leads to the constitution of the haplotypes presented in Fig. 2 and is the only explanation for the existing genotypes. We called the two loci DRB1 and DRB2, respectively. DRB1 is polymorphic with three alleles $\left(D R B^{*} 02,{ }^{*} 03\right.$, $\left.{ }^{*} 04\right)$, and DRB2 is polymorphic for the absence/presence of $D R B^{*} 01$.

$D R B^{*} 01$ is not detectable by PCR-SSCP/HD and sequencing at the $\mathrm{CDNA}$ level. Additionally, direct sequencing of cDNA-PCR products from $D R B^{*} 01^{*} 02$ and $D R B^{*} 01^{*} 03$ homozygous individuals gave clear sequences, while genomic DNA-derived PCR products gave mixed sequences because of the presence of $D R B^{*} 01$. Thus, $D R B^{*} 01$ was not expressed and so we further reduced our considerations to the locus DRB1 and its three alleles $D R B^{*} 02,{ }^{*} 03$ and ${ }^{*} 04$.

We found $D R B^{*} 02$ in $50, D R B^{*} 03$ in 72 and $D R B^{*} 04$ in 72 animals. The pairwise nucleotide divergence ranged between 16 and 38 nucleotides in the $171 \mathrm{bp}$ fragment (pairwise amino acid sequence divergences given in Table. 1). The ratio of nonsynonymous to synonymous substitutions $\left(\mathrm{d}_{\mathrm{N}} / \mathrm{d}_{\mathrm{S}}\right)$ was $0.39(P=0.01)$ in non-antigen-binding sites and $1.09(P=0.81)$ in antigen-binding sites according to Brown et al. (1993). At the population level, there was a slight deviation from Hardy-Weinberg equilibrium with more heterozygotes than expected $\left(H_{\text {exp }}=0.664, H_{\text {obs }}=0.717, P=0.012\right)$.

\section{Endoparasite load}

The subadults of the study population harboured three different species of nematodes in the gastro-intestinal tract and (as assessed by faecal oocyte counts) several species of coccidia in the gut (Eimeria spp.) and the liver (Eimeria stiedai). The distribution of the loads with the different endoparasites was right-skewed with the majority of the animals having rather low and only few individuals showing extremely high infestations.

The median number of the three nematodes in the gastro-intestinal tracts of the young rabbits (given as total number per individual) was 8110 , ranging from 180 to 105500 . The median number of G. strigosum found in the stomach was 100 (min: 5; max: 940). The median number of $P$. ambiguus occurring in the duodenum and in the colon was 7340 (min: 0; max: 103 920), and the median number of $T$. retortaeformis, which was found in the small intestine, was 390 (min: 0; max: 3330 ).

The number of coccidia oocytes (Eimeria spp.) per gram of faeces ranged between 130 and 416 150, and the median value was 6930 . The median number of oocytes of the species $E$. stiedai, which typically infests the liver, was 1380 (min: 10; max: 411 980), and the median number of oocytes from gut-colonizing Eimeria species was 3560 (min: 120; max: 338 270).

\section{Age and sex effects on endoparasite load}

Endoparasite loads with the different species or groups of species of nematodes and coccidia were significantly correlated with the age of the hosts (Table 2). These cor-

Table 1 Distribution and constitution of the different genotypes occurring in our sample $(n=113)$ of juvenile rabbits

\begin{tabular}{|c|c|c|c|c|c|c|c|}
\hline & \multirow[b]{2}{*}{$n$} & \multicolumn{3}{|c|}{ Locus DRB1 } & \multirow{2}{*}{$\frac{\text { Locus DRB2 }}{D R B^{*} 01}$} & \multirow{2}{*}{$\begin{array}{l}\text { Allele } \\
\text { divergence } \\
\text { (overall) }\end{array}$} & \multirow{2}{*}{$\begin{array}{l}\text { Allele } \\
\text { divergence } \\
\text { (PBR) }\end{array}$} \\
\hline & & $D R B^{*} 02$ & $D R B^{*} 03$ & $D R B^{*} 04$ & & & \\
\hline Genotype ${ }^{*} 02 /{ }^{*} 02$ & 12 & + & & & + & 0 & 0 \\
\hline Genotype ${ }^{*} 03 /{ }^{*} 03$ & 9 & & + & & + & 0 & 0 \\
\hline Genotype ${ }^{*} 04 /{ }^{*} 04$ & 11 & & & + & & 0 & 0 \\
\hline Genotype ${ }^{*} 02 /{ }^{*} 03$ & 20 & + & + & & + & 17 & 7 \\
\hline Genotype *02/*04 & 18 & + & & + & + & 18 & 8 \\
\hline Genotype ${ }^{*} 03 /{ }^{*} 04$ & 43 & & + & + & + & 8 & 3 \\
\hline
\end{tabular}

Pairwise allele divergence of the whole $171 \mathrm{bp}$ fragment and at the peptide-binding region (PBR) according to Brown et al. (1993) is given as the number of amino acid differences of the two alleles at the expressed locus DRB1. DRB2 represents a second locus that is polymorphic for absence and presence of an allele but is not expressed. Genotypes 1-3 are homozygous; genotypes 4-6 are heterozygous. 
Table 2 Linear mixed effects models for the effects of the presence/absence of different MHC class II DRB alleles and of MHC heterozygosity on the loads with different species of (a) endoparasitic nematodes and (b) coccidia in subadult European rabbits, including the effects of age, sex and year

\begin{tabular}{|c|c|c|c|c|c|}
\hline & Predictor variables & Estimate & $95 \%$ lower & $95 \%$ upper & $P$ \\
\hline \multicolumn{6}{|l|}{ (a) } \\
\hline \multirow[t]{7}{*}{ Nematoda (total) } & Sex & -0.384 & -0.889 & 0.137 & 0.13 \\
\hline & Age & 0.013 & 0.005 & 0.020 & $<0.001$ \\
\hline & Year & 0.812 & 0.247 & 1.434 & 0.055 \\
\hline & Heterozygosity & -0.182 & -0.875 & 0.467 & 0.59 \\
\hline & $D R B^{*} 02$ & 0.159 & -0.555 & 0.879 & 0.67 \\
\hline & $D R B^{*} 03$ & 0.050 & -0.591 & 0.687 & 0.89 \\
\hline & $D R B^{*} 04$ & -0.172 & -0.833 & 0.500 & 0.61 \\
\hline \multirow[t]{7}{*}{ Graphidium strigosum } & Sex & 0.267 & 0.009 & 0.517 & 0.040 \\
\hline & Age & 0.005 & 0.001 & 0.008 & 0.009 \\
\hline & Year & 0.304 & -0.055 & 0.649 & 0.91 \\
\hline & Heterozygosity & 0.032 & -0.306 & 0.356 & 0.87 \\
\hline & $D R B^{*} 02$ & -0.027 & -0.409 & 0.330 & 0.88 \\
\hline & $D R B^{*} 03$ & 0.039 & -0.283 & 0.370 & 0.83 \\
\hline & $D R B^{*} 04$ & -0.131 & -0.455 & 0.237 & 0.45 \\
\hline \multirow[t]{7}{*}{ Passalurus ambiguus } & Sex & -2.370 & -4.511 & -0.205 & 0.033 \\
\hline & Age & 0.087 & 0.057 & 0.116 & $<0.001$ \\
\hline & Year & 2.273 & -0.628 & 5.333 & 0.14 \\
\hline & Heterozygosity & 0.630 & -2.027 & 3.371 & 0.66 \\
\hline & $D R B^{*} 02$ & 0.426 & -2.639 & 3.565 & 0.79 \\
\hline & $D R B^{*} 03$ & 0.643 & -2.113 & 3.407 & 0.65 \\
\hline & $D R B^{*} 04$ & -0.254 & -3.021 & 2.797 & 0.86 \\
\hline \multirow[t]{7}{*}{ Trichostrongylus retortaeformis } & Sex & 0.177 & -0.231 & 0.583 & 0.39 \\
\hline & Age & -0.013 & -0.019 & -0.008 & $<0.001$ \\
\hline & Year & 1.128 & 0.587 & 1.681 & $<0.001$ \\
\hline & Heterozygosity & -0.061 & -0.555 & 0.462 & 0.81 \\
\hline & $D R B^{*} 02$ & 0.396 & -0.593 & 0.955 & 0.17 \\
\hline & $D R B^{*} 03$ & -0.060 & -0.566 & 0.434 & 0.82 \\
\hline & $D R B^{*} 04$ & -0.027 & -0.019 & 0.515 & 0.92 \\
\hline \multicolumn{6}{|l|}{ (b) } \\
\hline \multirow[t]{7}{*}{ Coccidia (total) } & Sex & 0.398 & -0.071 & 0.895 & 0.11 \\
\hline & Age & -0.013 & -0.019 & -0.006 & $<0.001$ \\
\hline & Year & -0.827 & -1.513 & -0.169 & 0.018 \\
\hline & Heterozygosity & -0.249 & -0.878 & 0.362 & 0.43 \\
\hline & $D R B^{*} 02$ & -0.361 & -0.977 & 0.252 & 0.24 \\
\hline & $D R B^{*} 03$ & -0.349 & -0.894 & 0.344 & 0.43 \\
\hline & $D R B^{*} 04$ & -0.789 & -1.449 & -0.127 & 0.019 \\
\hline \multirow[t]{7}{*}{ Hepatic coccidia (Eimeria stiedai) } & Sex & 0.667 & 0.116 & 1.198 & 0.014 \\
\hline & Age & -0.016 & -0.023 & -0.009 & $<0.001$ \\
\hline & Year & -1.417 & -2.153 & -0.668 & $<0.001$ \\
\hline & Heterozygosity & -0.234 & -0.900 & 0.453 & 0.49 \\
\hline & $D R B^{*} 02$ & -0.546 & -1.320 & 0.212 & 0.15 \\
\hline & $D R B^{*} 03$ & -0.233 & -0.910 & 0.453 & 0.51 \\
\hline & $D R B^{*} 04$ & -1.101 & -1.789 & -0.377 & 0.002 \\
\hline \multirow[t]{7}{*}{ Coccidia in the gut (Eimeria spp.) } & Sex & 0.138 & -0.388 & 0.654 & 0.60 \\
\hline & Age & -0.008 & -0.015 & -0.001 & 0.031 \\
\hline & Year & -0.283 & -1.021 & 0.432 & 0.44 \\
\hline & Heterozygosity & -0.187 & -0.874 & 0.455 & 0.58 \\
\hline & $D R B^{*} 02$ & -0.308 & -1.075 & 0.418 & 0.42 \\
\hline & $D R B^{*} 03$ & -0.194 & -0.862 & 0.500 & 0.57 \\
\hline & $D R B^{*} 04$ & -0.513 & -1.209 & 0.200 & 0.15 \\
\hline
\end{tabular}

Data derive from 113 animals from 2 years; animals were always sampled in mid November. Litter identity was included as a random factor. $P$-values and parameter estimates (including $95 \%$ confidence intervals) were calculated by $10000 \mathrm{Markov}$-chain Monte Carlo simulation runs. None of the tested 2-way interactions were significant $(P>0.10)$ and were omitted before re-calculating the models. A further backward elimination of nonsignificant predictors (not shown) did not lead to different results than obtained by calculating $P$-values for the full model including all main effects. 

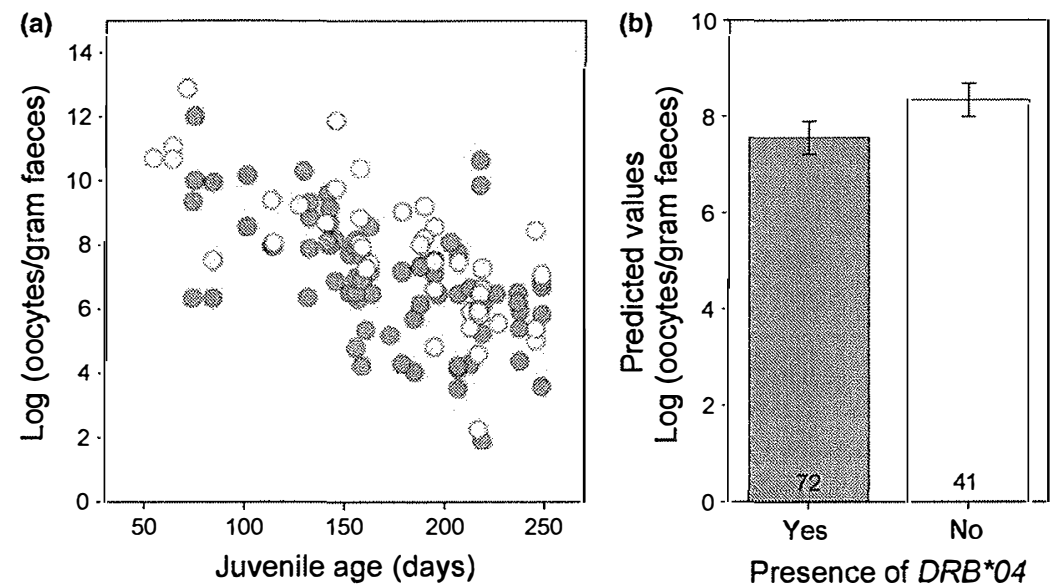

Fig. 3 (a) Correlation between the log-transformed load with hepatic coccidia oocytes and the age of juvenile European rabbits

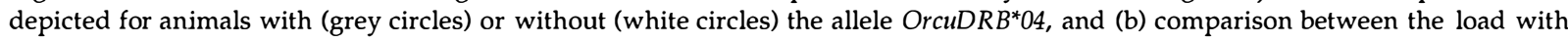
liver coccidia in juveniles (predicted values $\pm \mathrm{SE}$; sex, age and year are set constant) with or without the allele OrcuDRB*04. Differences are significant, see Table 2 for statistics.

relations were positive, i.e. the parasite loads increased with the rabbits' age for the nematode species $P$. ambiguus and $G$. strigosum, but were negative for T. retortaeformis and for all groups of coccidia. We also found significant effects of sex on the load with $E$. stiedai with young males having higher infestations than females. In addition, the parasite loads with $T$. retortaeformis and E. stiedai differed significantly between years.

There were no significant interactions of either year or sex with the occurrence of the different alleles $(P>0.10)$ or with MHC heterozygosity $(P>0.10)$, suggesting that the effects of MHC constitution on parasite load described in the following sections were independent of year and sex.

\section{Effects of MHC heterozygosity and occurrence of specific alleles}

Multivariate analyses revealed that young rabbits carrying the allele $\operatorname{OrcuDRB}{ }^{*} 04$ showed significantly lower infestations with coccidia (as assessed by the lower excretion rate of oocytes) than animals lacking this allele (Table 2b, Fig. 3b). More specifically, this difference was apparent with respect to infestations with coccidia occurring in the liver (E. stiedai) but was not seen with respect to Eimeria species occurring in the gut. Please note that this difference was still significant after Bonferroni correction, considering the multiple comparisons of the three different alleles $\left(\alpha_{\text {corr. }}=0.017\right)$. The occurrence of the alleles $D R B^{*} 02$ and $D R B^{*} 03$ as well as heterozygosity did not affect the load with any of the endoparasite species considered (Table 2).

Note that the differences in the load with hepatic coccidia oocytes between animals with and without
$D R B^{*} 04$ were not statistically significant when the age of the animals was not considered in the analysis $(P=0.13)$. This suggests that the effects were obviously masked by the strong age dependence of the number of oocytes per gram of faeces (see Fig. 3a, Table $2 b$ ).

\section{Differences among MHC genotypes}

We also found differences with respect to the loads with hepatic coccidia when considering the different genotypes as a factor with six levels ( $P=0.012$; Fig. 4). Here also, we found significant effects of age $(P<0.001)$, sex $(P=0.010)$ and year $(P<0.001)$. Post hoc analysis revealed that the two heterozygous genotypes with allele $D R B^{*} 04$ predominantly had lower loads than the other four genotypes (although $P$-values of the post hoc comparisons between genotype ${ }^{*} 02 /{ }^{*} 04$ and ${ }^{*} 02 /^{*} 02$ or ${ }^{*} 03 /{ }^{*} 03$ barely missed the $5 \%$ level of significance). The parasite load did not differ significantly between these two genotypes (post hoc statistics in Fig. 4). And again, the MHC-related differences between the different genotypes were not statistically significant when the age of the animals was removed from the model $(P=0.084)$.

We systematically compared the loads with all other groups of parasites (as given in Table 2) among young rabbits with different genotypes. However, there were no significant differences with respect to any other response variable $(P>0.15)$.

\section{Discussion}

In this study, we analysed the influence of individual MHC class II DRB constitution on endoparasite load in 


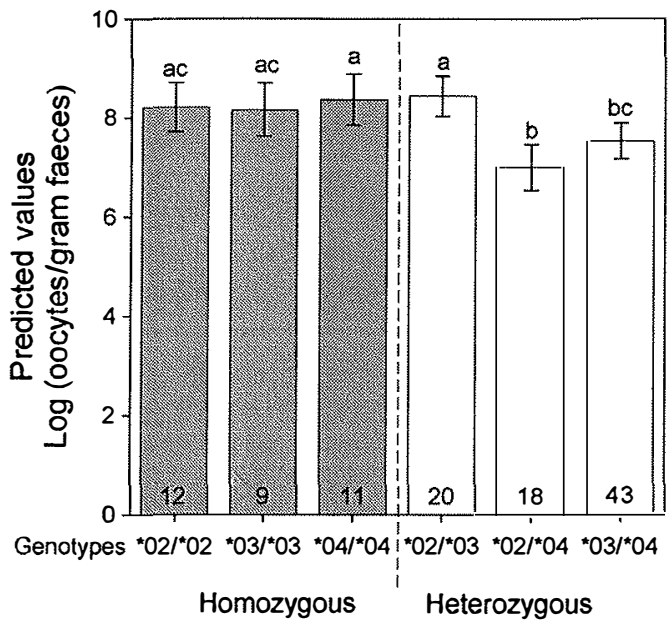

Fig. 4 Comparison between the log-transformed load with hepatic coccidia oocytes (predicted values $\pm \mathrm{SE}$; sex, age and year are set constant) of young rabbits with different MHC class II DRB genotypes (different homozygous and heterozygous combinations of $\operatorname{OrcuDRB}{ }^{*} 02,{ }^{*} 03$ and ${ }^{*} 04$ ). Significant differences between groups are denoted by different letters $(P<0.05)$; see text for details of statistics.

subadult European rabbits. Within an isolated population, we identified three expressed MHC DRB alleles forming three heterozygous and three homozygous genotypes. As expected (Stodart 1968; Gallazzi 1977; Cattadori et al. 2005; Cornell et al. 2008), we found agespecific differences in infestations of the young rabbits with different endoparasite species. Furthermore and as it has been published previously (Lochmiller \& Deerenberg 2000; Soler 2003), males showed higher infestations than females with respect to some of the studied endoparasite species.

Correcting for age, sex and year of sampling, we found an association between the allele $D R B^{*} 04$ and a lowered infestation with hepatic coccidia (E. stiedai), but no general heterozygote advantage. Such an association of specific MHC alleles with the occurrence of certain pathogens is generally discussed as an indication, hence not direct evidence for frequency-dependent selection (Sommer 2005). However, a closer look at the genotypes revealed a more complex pattern. Of the six genotypes, two $\left(D R B^{*} 02 /^{*} 04\right.$ and $\left.{ }^{*} 03 /{ }^{*} 04\right)$ were advantageous with respect to hepatic coccidia. Both are heterozygous and both contain the allele $D R B^{*} 04$. If the advantage was the result of the possession of $D R B^{*} 04$, then it should also be provided by $D R B^{*} 04 /{ }^{*} 04$ homozygous genotypes Otherwise, if the advantage was the result of heterozygosity per se, also the heterozygous combination $D R B^{*} 02 /^{*} 03$ should be beneficial. Neither was the case. Finally, if the advantage was the result of an additive effect of hetero- zygosity and of the possession of $D R B^{*} 04$, then we would expect at least intermediate effects in ${ }^{*} 02 /{ }^{*} 03$ and ${ }^{*} 04{ }^{*} 04$ genotypes. This was also not the case, and thus the apparent result of a general advantage of the allele $D R B^{*} 04$ is misleading. Our study rather provides evidence for heterozygote superiority (i.e. advantage of a particular heterozygous genotype over the corresponding homozygotes, McClelland et al. 2003) of genotypes containing $D R B^{*} 04$ emerging in a nonadditive manner from the combination of this allele with any of the two other alleles.

Thus, not all but only certain heterozygous combinations provided an advantage. The question that arises here is what accounts for the quality of these combinations? One explanation may be an advantage for heterozygotes only with divergent alleles (Wakeland et al. 1990), whereas heterozygous combinations of similar alleles and thus functionally similar MHC products might feature a peptide-binding repertoire similar to that of homozygotes. The adaptive value of different genotypes may therefore vary because of different divergence. We compared divergence of the peptidebinding region between the three heterozygous genotypes. The two advantageous genotypes have an allelic divergence of three and eight amino acid residues of the peptide-binding region (15 sites), while the disadvantageous one has an allele divergence of seven amino acid residues. Obviously, this does not explain the advantage of the former genotypes. Generally, we propose that it may be critical to consider traits of a single locus within such a dense cluster of genes with similar function as independent from the surrounding genes. Instead, a single gene should rather serve as a marker for the whole MHC haplotype, which contains five alpha-chain genes (DRA, DQA, DPA1, DPA2, DNA, LeGuern et al. 1985) and at least seven polymorphic beta-chain genes (DRB1 to DRB5, DQB, DPB, Sittisombut et al. 1989) within the rabbit class II region. We suggest rather taking into account heterozygosity and functional divergence of all these loci to get an adequate measure of functional divergence. A detailed analysis of allele divergence on a single MHC gene would only make sense if this locus would be unique and not subject to duplication, as has been found in the class II region of many salmonids. Here, allele divergence seems to be an important mechanism in different aspects of MHC function (Landry et al. 2001; Consuegra \& de Leaniz 2008; Neff et al. 2008). Interestingly, in our study population, the two advantageous MHC genotypes with respect to the load with hepatic coccidia are the ones that must be heterozygous at the DRB2 locus, which is polymorphic for the absence and presence of the pseudoallele OrcuDRB*01. Even if this gene is not expressed, individuals being homozygous at this 
locus might also be homozygous at some linked loci, providing some degree of disadvantage.

Only recently, examples demonstrating heterozygote superiority have been reported in studies of Arctic char (Salvelinus alpinus, Kekäläinen et al. 2009) and water voles (Arvicola terrestris, Oliver et al. 2009). Both studies are based on populations with two alleles forming three possible genotypes, which is the maximally reduced polymorphism. In both studies, the only heterozygous genotype was superior to the two homozygous genotypes with respect to parasite load and other fitnesscorrelated traits. Our study represents a three-allele system, consequently forming six genotypes. This allows the emergence of more complex patterns, such as the here reported differences in performance of the three occurring heterozygous genotypes (Fig. 4).

It remains open why we only found a relationship between MHC constitution and the load with hepatic coccidia, but not with other coccidia (Eimeria) species or nematodes infesting the gut. Generally, immune reactions against both Eimeria and nematodes are dependent on the cellular adaptive immune response (nematodes: Else 2005; Eimeria: Pakandl 2009), and relationships between MHC constitution and nematode load have also been described in some species (e.g. Paterson et al. 1998; Axtner \& Sommer 2007). On the one hand, such specific correlations between MHC constitution and the loads with particular endoparasite species may arise from slight differences in the immune response to infestations with different groups of endoparasites. On the other hand, nonsignificant correlations are difficult to interpret and might not necessarily signify the lack of a causal relationship. The consideration of other potentially confounding environmental variables such as weather conditions (Stodart 1968; O'Connor et al. 2006) or an animal's social environment (von Holst 2001) may help to detect even small effects of the MHC constitution on an animal's susceptibility to specific parasites.

Although European rabbit populations today are primarily limited by viral diseases such as myxomatosis and rabbit haemorrhagic disease (Mutze et al. 2002; Moreno et al. 2007), hepatic coccidiosis is considered a significant contributor to juvenile mortality (Mykytowycz 1961; Cowan 1987; Singla et al. 2000). Thus, the observed relationship between MHC constitution and the infestation with hepatic coccidia has important implications for the individual. Although the young rabbits of our study had already survived the most critical phase of hepatic coccidiosis, which is mainly during the first few weeks after weaning, highly infested animals could serve as potential sources leading to severe infections of later born young (Wang \& Tsai 1991). Furthermore, even if the animals survive to maturity, parasite infections can limit growth, development and fecundity and thus have the potential to exert long-term consequences on fitness-correlated traits (Lindström 1999; Lummaa \& Clutton-Brock 2002; Hakkarainen et al. 2007; Vandegrift et al. 2008).

In conclusion, our study demonstrates heterozygote superiority of an MHC class II gene with respect to hepatic coccidiosis, but only with respect to specific heterozygous genotypes. Furthermore, we could show that the classical approach of parasite-MHC allele association studies can result in misleading findings.

\section{Acknowledgements}

We thank all the students who helped with the field work. We are also grateful to Jon Slate and three anonymous reviewers for their helpful comments and to Robyn Hudson, UNAM, México, for checking the English. The study was financially supported by a grant from the German Research Foundation DFG (Ro 2431/3-1).

\section{References}

Apanius V, Penn D, Slev PR, Ruff LR, Potts WK (1997) The nature of selection on the major histocompatibility complex. Critical Reviews in Immunology, 17, 179-224.

Arkush KD, Giese AR, Mendonca HL et al. (2002) Resistance to three pathogens in the endangered winter-run chinook salmon (Oncorhynchus tshawytscha): effects of inbreeding and major histocompatibility complex genotypes. Canadian Journal of Fisheries and Aquatic Sciences, 59, 966-975.

Axtner J, Sommer S (2007) Gene duplication, allelic diversity, selection processes and adaptive value of MHC class II DRB genes of the bank vole, Clethrionomys glareolus. Immunogenetics, 59, 417-426.

Baayen RH, Davidson DJ, Bates DM (2008) Mixed-effects modeling with crossed random effects for subjects and items. Journal of Memory and Language, 59, 390-412.

Babik W, Durka W, Radwan J (2005) Sequence diversity of the MHC DRB gene in the Eurasian beaver (Castor fiber). Molecular Ecology, 14, 4249-4257.

Bates D (2005) Fitting linear mixed models in R. R News, 5, 27 39.

Brown JH, Jardetzky TS, Gorga JC et al. (1993) Three-dimensional structure of the human class II histocompatibility antigen HLA-DR1. Nature, 364, 33-39.

Budowle B, Chakraborty R, Giusti AM, Eisenberg AI, Allen RC (1991) Analysis of the VNTR locus D1S80 by the PCR followed by high-resolution PAGE. American Journal of Human Genetics, 48, 137-144.

Cattadori IM, Boag B, Bjørnstad ON, Cornell SJ, Hudson PJ (2005) Peak shift and epidemiology in a seasonal hostnematode system. Proceedings of the. Royal Society B: Biological Sciences, 272, 1163-1169.

Consuegra S, de Leaniz G (2008) MHC-mediated mate choice increases parasite resistance in salmon. Proceedings of the Royal Society B: Biological Sciences, 275, 1397-1403.

Corbet GB (1994) Taxonomy and origins. In:The European Rabbit. The History and Biology of a Successful Colonizer (eds 
Thomson HV, King CM), pp. 1-6. Oxford University Press, Oxford.

Cornell SJ, Bjørnstad ON, Cattadori IM, Boag B, Hudson PJ (2008) Seasonality, cohort-dependence and the development of immunity in a natural host-nematode system. Proceedings of the Royal Society B: Biological Sciences, 275, 511-518.

Cowan DP (1987) Patterns of mortality in a free-living rabbit (Oryctolagus cuniculus) population. Symposium of the Zoological Society London, 58, 59-77.

Croisetière S, Tarte PD, Bernatchez L, Belhumeur P (2008) Identification of MHC class IIB resistance/susceptibility alleles to Aeromonas salmonicida in brook charr (Salvelinus fontinalis). Molecular Immunology, 45, 3107-3116.

Ditchkoff SS, Lochmiller RL, Masters RE, Hoofer SR, van den Bussche RA (2001) Major-histocompatibility-complexassociated variation in secondary sexual traits of white-tailed deer (Odocoileus virginianus): evidence for good-genes advertisement. Evolution, 55, 616-625.

Doherty PC, Zinkernagel RM (1975) A biological role for the major histocompatibility antigens. Lancet, 1, 1406-1409.

Donald AD, Dineen JK, Symons LEA (1982) Biology and Control of Endoparasites: McMaster Laboratory 50th Anniversary Symposium in Parasitology Held at the University of Sydney 5-6 November 1981. Academic Press, Sydney.

Else KJ (2005) Have gastrointestinal nematodes outwitted the immune system? Parasite Immunology, 27, 407-416.

Excoffier L, Laval G, Schneider S (2005) Arlequin (version 3.0): an integrated software package for population genetics data analysis. Evolutionary Bioinformatics Online, 1, 4750.

Fain MA, Zhao T, Kindt TJ (2001) Improved typing procedure for the polymorphic single-copy RLA-DQA gene of the rabbit reveals a new allele. Tissue Antigens, 57, 332-338.

Faraway JJ (2006) Extending the Linear Model with R: Generalized Linear, Mixed Effects and Nonparametric Regression Models. Chapman \& Hall/CRC.

Fox J, Monette G (1992) Generalized collinearity diagnostics. Journal of the American Statistical Association, 87, 178-183.

Froeschke G, Sommer S (2005) MHC class II DRB variability and parasite load in the striped mouse (Rhabdomys pumilio) in the Southern Kalahari. Molecular Biology and Evolution, 22, 1254-1259.

Gallazzi D (1977) Cyclical variations in the excretion of intestinal coccidial oocysts in the rabbit. Folia Veterinaria Latina, 7, 371-380.

Gibb JA (1979) Factors affecting population density in the wild rabbit, Oryctolagus cuniculus (L.), and their relevance to small mammals. In:Evolutionary Ecology (eds Stonehouse B, Perrins C). pp. 33-46, MacMillan, London.

Gordon HM, Whitlock HV (1939) A new technique for counting nematode eggs in sheep faeces. Journal of the Council for Scientific and Industrial Research (Australia), 12, 5052.

Goüy de Bellocq BJ, Suchentrunk F, Baird SJ, Schaschl H (2008) Evolutionary history of an MHC gene in two leporid species: characterisation of Mhc-DQA in the European brown hare and comparison with the European rabbit. Immunogenetics, 61, 131-144.

Guo SW, Thompson EA (1992) Performing the exact test of Hardy-Weinberg proportion for multiple alleles. Biometrics, $48,361-372$.
Hakkarainen H, Huhta E, Koskela E et al. (2007) Eimeriaparasites are associated with a lowered mother's and offspring's body condition in island and mainland populations of the bank vole. Parasitology, 134, 23-31.

Hall TA (1999) BioEdit: a user-friendly biological sequence alignment editor and analysis program for Windows 95/98/NT. Nucleic Acids Symposium Series, 41, 95-98.

Harf R, Sommer S (2005) Association between MHC class II DRB alleles and parasite load in the hairy-footed gerbil, Gerbillurus paeba, in the Southern Kalahari. Molecular Ecology, 14, 85-91.

Hobbs RP, Twigg LE, Elliot AD, Wheeler AG (1999) Evaluation of the association of parasitism with mortality of wild European rabbits Oryctolagus cuniculus (L.) in southwestern Australia. The Journal of Parasitology, 85, 803-808.

von Holst D (2001) Social stress in wild mammals in their natural habitat. In:Coping with Challenge: Welfare in Animals Including Humans (ed Broom DM). pp. 317-335, University Press, Berlin, 317-335.

von Holst D, Hutzelmeyer H, Kaetzke P et al. (2002) Social rank, fecundity and lifetime reproductive success in wild European rabbits (Oryctolagus cuniculus). Behavioral Ecology and Sociobiology, 51, 245-254.

Ilmonen P, Penn DJ, Damjanovich K et al. (2007) Major histocompatibility complex heterozygosity reduces fitness in experimentally infected mice. Genetics, 176, 25012508.

Janeway CA, Travers P, Walport M, Shlomchik M (2001) Immunobiology. Garland Science, New York and London.

Kekäläinen J, Vallunen JA, Primmer CR, Rättyä J, Taskinen J (2009) Signals of major histocompatibility complex overdominance in a wild salmonid population. Proceedings of the Royal Society B: Biological Sciences, 276, 3133-3140.

Kessel JF, Jankiewicz A (1931) Species differentiation of the coccidia of the domestic rabbit based on a study of oocysts. American Journal of Epidemiology, 14, 304-324.

Klein J, Bontrop RE, Dawkins RL et al. (1990) Nomenclature for the major histocompatibility complexes of different species: a proposal. Immunogenetics, 31, 217-219.

Kotlan A, Pellerdy L (1949) A survey of the species of Eimeria occurring in the domestic rabbit. Acta Veterinaria Academiae Scientiarum Hungaricae, 1, 93-97.

Landry C, Garant D, Duchesne P, Bernatchez L (2001) 'Good genes as heterozygosity': the major histocompatibility complex and mate choice in Atlantic salmon (Salmo salar). Proceedings of the Royal Society of London, Series B: Biological Sciences, 268, 1279-1285.

LeGuern C, Marche PN, Kindt TJ (1985) Molecular evidence for five distinct MHC class II genes in the rabbit. Immunogenetics, 22, 141-148.

Lindström J (1999) Early development and fitness in birds and mammals. Trends in Ecology \& Evolution, 14, 343-348.

Lochmiller RL, Deerenberg C (2000) Trade-offs in evolutionary immunology: just what is the cost of immunity? Oikos, 88, 87-98.

Lummaa V, Clutton-Brock T (2002) Early development, survival and reproduction in humans. Trends in Ecology $\mathcal{E}$ Evolution, 17, 141-147.

McClelland EE, Penn DJ, Potts WK (2003) Major histocompatibility complex heterozygote superiority during coinfection. Infection and Immunity, 71, 2079-2086. 
Moreno S, Beltran JF, Cotilla I et al. (2007) Long-term decline of the European wild rabbit (Oryctolagus cuniculus) in southwestern Spain. Wildlife Research, 34, 652-658.

Mutze G, Bird P, Kovaliski J et al. (2002) Emerging epidemiological patterns in rabbit haemorrhagic disease, its interaction with myxomatosis, and their effects on rabbit populations in South Australia. Wildlife Research, 29, 577590.

Mykytowycz R (1956) A survey of endoparasites of the wild rabbit, Oryctolagus cuniculus (L.) in Australia. Wildlife Research, 1, 19-25.

Mykytowycz R (1961) Social behaviour of an experimental colony of wild rabbits, Oryctolagus cuniculus (L.). IV. Conclusion: outbreak of myxomatosis, third breeding season, and starvation. Wildlife Research, 6, 142-155.

Nagelkerke NJD (1991) A note on a general definition of the coefficient of determination. Biometrika, 78, 691-692.

Neff BD, Garner SR, Heath JW, Heath DD (2008) The MHC and non-random mating in a captive population of Chinook salmon. Heredity, 101, 175-185.

Nei M, Gojobori T (1986) Simple methods for estimating the numbers of synonymous and nonsynonymous nucleotide substitutions. Molecular Biology and Evolution, 3, 418425.

O'Connor LJ, Walkden-Brown SW, Kahn LP (2006) Ecology of the free-living stages of major trichostrongylid parasites of sheep. Veterinary Parasitology, 142, 1-15.

Oliver MK, Piertney SB (2006) Isolation and characterization of a MHC class II DRB locus in the European water vole (Arvicola terrestris). Immunogenetics, 58, 390-395.

Oliver MK, Telfer S, Piertney SB (2009) Major histocompatibility complex (MHC) heterozygote superiority to natural multi-parasite infections in the water vole (Arvicola terrestris) Proceedings of the Royal Society B: Biological Sciences, 276, 1119-1128.

Pakandl M (2009) Coccidia of rabbit: a review. Folia Parasitologica, 56, 153-166.

Paterson S, Wilson K, Pemberton JM (1998) Major histocompatibility complex variation associated with juvenile survival and parasite resistance in a large unmanaged ungulate population (Ovis aries L.). Proceedings of the National Academy of Sciences of the United States of America, 95, 37143719.

$\mathrm{R}$ Development Core Team (2009) R: A Language and Environment for Statistical Computing. $\mathrm{R}$ Foundation for Statistical Computing, Vienna, Austria; ISBN 3-900051-07-0, URL http://www.R-project.org.

Richardson BJ, Wood DH (1982) Experimental ecological studies on a subalpine rabbit population. I. Mortality factors acting on emergent kittens. Wildlife Research, 9, 443450.

Richman AD, Herrera LG, Nash D (2003) Evolution of MHC class II EB diversity within the genus Peromyscus. Genetics, 164, 289-297.

Rödel HG, Bora A, Kaetzke P et al. (2004) Over-winter survival in subadult European rabbits: weather effects, density dependence, and the impact of individual characteristics. Oecologia, 140, 566-576.

Rödel HG, Hudson R, von Holst D (2008a) Optimal litter size for individual growth of European rabbit pups depends on their thermal environment. Oecologia, 155, 677-689.
Rödel HG, Starkloff A, Bruchner B, von Holst D (2008b) Social environment and reproduction in female European rabbits (Oryctolagus cuniculus): benefits of the presence of litter sisters. Journal of Comparative Psychology, 122, 73-83.

Rödel HG, von Holst D, Kraus C (2009) Family legacies: shortand long-term fitness consequences of early-life conditions in female European rabbits. Journal of Animal Ecology, 78, 789797.

Schad J, Sommer S, Ganzhorn JU (2004) MHC variability of a small lemur in the littoral forest fragments of southeastern Madagascar. Conservation Genetics, 5, 299-309.

Singla LD, Juyal PD, Sandhu BS (2000) Pathology and therapy in naturally Eimeria stiedae-infected rabbits. Journal of Protozoology Research, 10, 185-191.

Sittisombut N, Knight KL (1986) Rabbit major histocompatibility complex. I. Isolation and characterization of three subregions of class II genes. Journal of Immunology, 136, 18711875 .

Sittisombut N, Tissot RG, Knight KL (1989) Rabbit major histocompatibility complex: III. Multiple class II DRB genes and restriction frament length polymorphism of the class II $\alpha$ and $B$ genes. International Journal of Immunogenetics, 16 63-75.

Soler JJ (2003) Trade-off between immunocompetence and growth in magpies: an experimental study. Proceedings of the Royal Society B: Biological Sciences, 270, 241-248.

Sommer S (2005) The importance of immune gene variability (MHC) in evolutionary ecology and conservation. Frontiers in Zoology, 2, 16.

Spieker-Polet H, Sittisombut N, Yam PC, Knight KL (1989) Rabbit major histocompatibility complex. IV. Expression of major histocompatibility complex class II genes. Journal of Immunogenetics, 17, 123-132.

Stodart E (1968) Coccidiosis in wild rabbits, Oryctolagus cuniculus (L.), at four sites in different climatic regions in eastern Australia I. Relationship with the age of the rabbit. Australian Journal of Zoology, 16, 69-85.

Surridge AK, van derLooW, Abrantes J et al. (2008) Diversity and evolutionary history of the MHC DQA gene in leporids. Immunogenetics, 60, 515-525.

Takahata N, Nei M (1990) Allelic genealogy under overdominant and frequency-dependent selection and polymorphism of major histocompatibility complex loci. Genetics, 124, 967978.

Tamura K, Dudley J, Nei M, Kumar S (2007) MEGA4: molecular evolutionary genetics analysis (MEGA) software version 4.0. Molecular Biology and Evolution, 24, 1596-1599.

The MHC Sequencing Consortium (1999) Complete sequence and gene map of a human major histocompatibility complex. Nature, 401, 921-923.

Thursz MR, Thomas HC, Greenwood BM, Hill AVS (1997) Heterozygote advantage for HLA class-II type in hepatitis B virus infection. Nature Genetics, 17, 11-12.

Tollenaere C, Bryja J, Galan M et al. (2008) Multiple parasites mediate balancing selection at two MHC class II genes in the fossorial water vole: insights from multivariate analyses and population genetics. Journal of Evolutionary Biology, 21, 13071320.

Vandegrift KJ, Raffel TR, Hudson PJ (2008) Parasites prevent summer breeding in white-footed mice, Peromyscus leucopus. Ecology, 89, 2251-2258. 
Wakeland EK, Boehme S, She JX et al. (1990) Ancestral polymorphisms of MHC class II genes: divergent allele advantage. Immunologic Research, 9, 115-122.

Wang JS, Tsai SF (1991) Prevalence and pathological study on rabbit hepatic coccidiosis in Taiwan. Proceedings of the National Science Council, Republic of China. Part B, Life sciences, 15, 240-243.

Wedekind C, Walker M, Little TJ (2005) The course of malaria in mice: major histocompatibility complex (MHC) effects, but no general MHC heterozygote advantage in single-strain infections. Genetics, 170, 1427-1430.

Westerdahl H, Waldenström J, Hansson B et al. (2005) Associations between malaria and MHC genes in a migratory songbird. Proceedings of the Royal Society B: Biological Sciences, 272, 1511-1518.
C.O's research focuses on the role of MHC genes in social behaviour and population genetics in mammals and fish. A.S. studies the relationship between environmental factors and endoparasite load in European rabbits. This research was part of her PhD thesis at the University of Bayreuth. P.R. studies are focused on host-parasite interactions. This research was part of his bachelor thesis at the University of Bayreuth. D.v.H.'s research focuses on the effects of social interactions on behaviour, fertility and health in mammals, mainly tree shrews and European rabbits. H.G.R. has longstanding interest in the social behaviour and ecology of European rabbits, and his research focuses on the impact of an animal's early developmental environment on fitness-related traits. 